\title{
Study on Reliability Evaluation Method Based on Improved Monte Carlo Method
}

\author{
Liu Jun ${ }^{1}$, Yang Fan $^{1}$, Ren Lijia ${ }^{2}$ \\ ${ }^{1}$ State Grid Shanghai Electric Power Research Institute, Shanghai, China \\ ${ }^{2}$ Shanghai University of Engineering Science, Shanghai, China
}

\begin{abstract}
The advancement in science and technology comes with continuously expanding power system scale, increasingly complex system operation condition and higher requirements for accuracy and speed of power system reliability evaluation, but actual calculation methods cannot meet the needs. Therefore, there is need to improve the reliability of conventional power distribution network so that requirements of calculation speed and calculation accuracy can be met. In this paper, reliability of the power distribution network will be evaluated using improved Monte Carlo method with uniform sampling. The average value is obtained through calculation of state of multiple sub-intervals and test functions, which effectively improves calculation accuracy, and further increases the utilization of random numbers. By improving the uniform sampling method, the Monte Carlo simulation variance is reduced, and evaluation and calculation efficiency is improved. At the same time, unqualified power grid is selected for analysis. Based on the simulation results, qualified power distribution networks are compared to point out where the requirements are not met. Also, comparative analysis is made on the effect of power distribution network grid structure etc. on the user's power supply. Finally, suggestions for improving power distribution network reliability are given from equipment reliability, grid structure.
\end{abstract}

\section{Introduction}

Power supply reliability means the ability of the power supply system to provide continuous power to the user. The interruption of power supply will not only incur huge economic losses, but also affect people's lives and social stability. High power supply reliability is not only what power consumers demand, but also what power supply companies pursue in self-development. Researches on reliability of power distribution network gradually began in the $1960 \mathrm{~s}$, originally as qualitative analysis. As the research deepens, pure qualitative analysis could not meet the research needs. Researchers adopted quantitative indicators to measure reliability. So far, the research progress has always been concerned by experts in related fields. In developed countries such as the United States, Japan and France, relevant agencies have been set up to analyze, research and plan power distribution networks, and reliability evaluation system has been relatively complete.

Commonly used reliability assessment methods can be categorized into analytical and simulation methods. The basic idea of analytical method is to build a corresponding model according to original reliability data of the components, and then obtain power grid reliability indicators through mathematical analysis. Analytical method features clear physical concept, high model precision, and clear logic concept. However, the computation burden increases sharply when component number increases, and direct use of analytical method faces difficulties when the power grid reaches a certain scale. Failure mode and effects analysis [1] (FMEA) and network methods are commonly used analytical methods. By using fault traversal algorithm, FMEA classifies component failures, determines the impact scope of the failure in combination with preorder first traversal and postorder first traversal methods. Its algorithm computation increases rapidly with the power distribution network increase [2-3]. Hence, many optimized algorithms are introduced based on analytical method, for instance, network methods based on topological structure of the power distribution system which include minimum path method [4-5], minimum cut-set method[6], network equivalent method[7], and region separation method[8].

Simulation method is recently introduced in the field of reliability analysis, which samples according to probability distribution of components, judges the state of components, and then obtains reliability-related indicators using statistical knowledge, mainly including Monte Carlo simulation method [9-10]. Simulation method, with intuitive and convenient characteristics, can well handle dynamic load variation. However, its accuracy is directly proportional to the consumed time. It often takes time to enable high accuracy in result, which is not conducive to targeted evaluation [11]. 
Computation speed means a lot for a good practical model and algorithm. This paper calculates and analyzes the actual power distribution network using improved Monte Carlo method of discrete sampling method, and studies measures to improve power supply reliability of power distribution network.

\section{Monte carlo simulation evaluation method}

Monte Carlo method is widely used in reliability evaluation. The specific idea is: First, the network is divided into components, and the failure probability of the grid is simulated according to the failure probability of each component. The grid state is determined by these components. Such a simulation constitutes an assessment of the grid to be simulated. Then, continue with this method, and set a certain amount of simulation as a sample. When enough data is collected, analysis can be made to obtain relevant indicators. Monte Carlo method supports evaluation of complex systems regardless of power distribution network structure. The number of samples required is also independent of the structure, so it can relatively well reflect some random factors. However, a large number of samples are required to obtain results close to the truth value, which will greatly increase the simulation time and affect the overall assessment as components show similar status.

\subsection{Ordinary sampling method}

In the conventional Monte Carlo method ${ }^{[43-48]}$, each component is divided into two states independent from each other and free from interference by other components. The state is generally set to 1 when the device is in normal operation and to 0 when in a fault state. The failure rate of the component is in the interval $[0,1]$. By generating a random number and comparing it with the failure rate of the component, the component state can be judged. State in which the primary grid is operated can be obtained by simulating each component and multiplying it with the probability function. Repeat the above steps constantly, and the average system reliability index can be calculated (generally estimated value due to the limitation of the sample number) when the sample reaches a certain size. The convergence criterion $\beta$ is then introduced, which concerns the expected variance of the reliability index and the variance of the component state function.

In order to meet the pre-set accuracy, Monte Carlo method requires a lot of simulation, which will sacrifice the operation time. On the contrary, guaranteeing simulation time demands reduced convergence accuracy as a price.

\subsection{Average and scattered sampling method}

The sampling method needs improvement for better speed and accuracy of Monte Carlo simulation. There are currently many effective improvement methods available. In this experiment, average and scattered sampling method is adopted to sample the operation state of the power system according to the following specific sampling steps:

(1) The interval $[0,1]$ is divided into $h$ intervals according to the maximum failure rate of the component in the system;

(2)A random number $a_{j}$ is generated for each element in the power system;

(3) If there is $k$ making $(k-1) / h<a_{j} \leq k / n+\beta_{j}\left(\beta_{j}\right.$ is the failure rate of component $j$ ), then component $j$ is faulty in interval $\mathrm{k}$ and in normal operation in other intervals ;

(4) The operation state of each component is averaged, and then operation state of each component is normal or with $1 / h$ failure probability, so the system operation status is obtained;

(5) Statistical analysis is made for the system operation status to determine whether the sample variance coefficient satisfies the convergence condition. Stop sampling if the condition is met. Otherwise, turn to step (2).

\section{3 the basic steps of Monte Carlo method to assess power system reliability}

According to the above theory, Monte Carlo method is mainly composed of three parts: system state sampling, system state analysis and system evaluation index statistics. The main steps of the assessment are shown in Figure 1 .

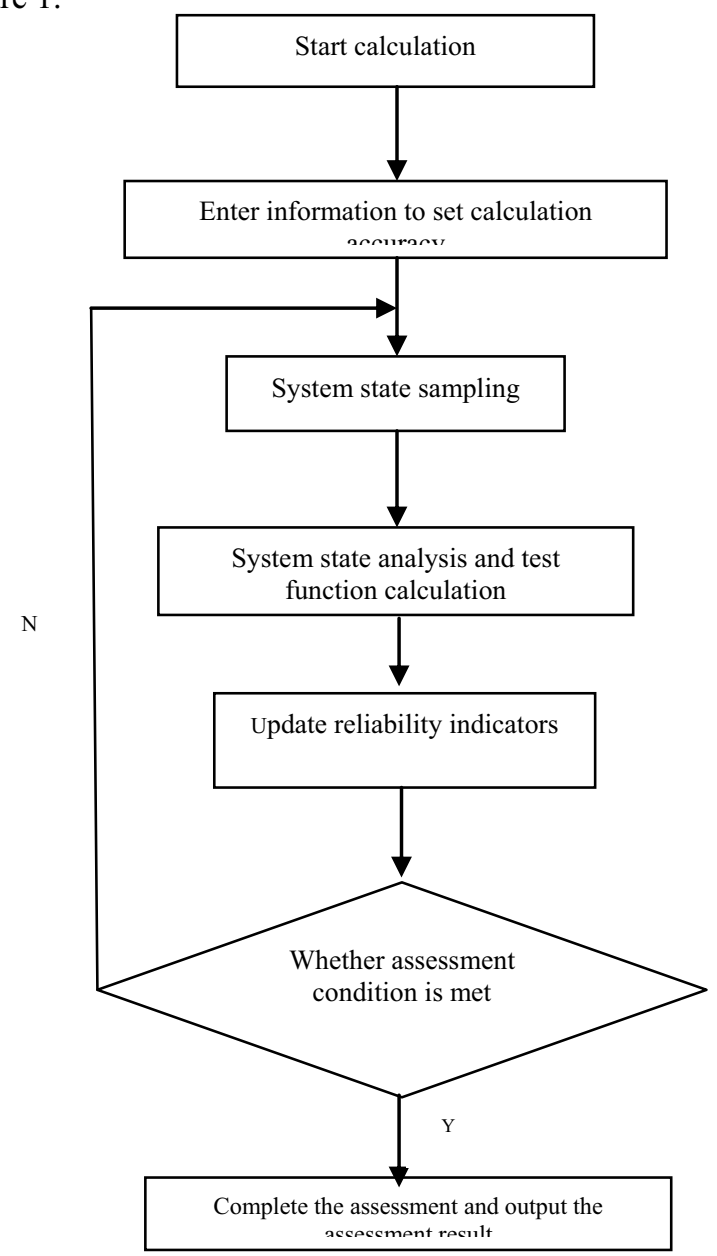

Fig. 1 Calculation Flow Chart of Monte Carlo Method 
It can be known from the above figure that Monte Carlo method need proceed with random state sampling of the system, without involving the duration and transfer rate of the state. The assessment process requires a higher degree of accuracy, so sampling algorithm can be improved during the sampling process to reduce the contrast and improve the calculation accuracy.

After completing the sampling of the system state, the test function value of the state needs to be analyzed and calculated. The test function calculation method corresponds to reliability evaluation index. State analysis of the power system mainly starts from two aspects: on the one hand, the power system state demands load flow analysis to judge fault existence in the system; on the other hand, the actual system state should be optimized based on load flow analysis to eliminate the fault state in the system and guarantee safe system operation. DC load flow analysis is a commonly used method for system optimization analysis.

\section{Reliability example analysis of power distribution network}

Reliability of actual power distribution network in a region is analyzed using dispersed sampling method such as the improved Monte Carlo described earlier. Figure 2 shows the power distribution network of a region. The power distribution network consists of 13 load points, 8 switches, 3 incoming lines, and 25 lines. The statistical data cover failure rate, repair time, load point location, user number, average load, line length, etc., specifically as follows:

Line failure rate of 25 lines: $0.0150($ times/year $\bullet \mathrm{km})$

Node failure rate: 0.065 (times/year $\bullet$ kilometers)

Line repair time: 5 (hours)

Node repair time: 20 (hours) 24

Load position: 2, 5, 6, 7, 11, 12, 13, 14, 15, 19, 20, 21,

User number at each load point: 147, 126, 1, 1, 132, $147,1,79,1,76,79,76,1$

Line length: $0.8,2.5,1.6,0.9,1.6,2.5,0.6,1.6,0.75$, $0.9,3.2,2.8,0.6,3.5,1.6,2.8,3.2,2.5,3.2,1.6,0.8,2.8$, $2.5,3.2,2.8(\mathrm{~km})$

The system component operation state is subject to average and scattered sampling. The component working status is judged and component repair time is simulated. Then, failure times and failure time of each load point are calculated according to the sampled system status. Whether experiment objective is reached is judged after each sampling calculation. Several experimental objectives were tried in this experiment, including sampling times, result variance and sampling time etc. The result variance should be taken as objective if there is a high requirement for result accuracy. The sampling times or time should be taken as objective if there is high requirement for system operation time. Finally, the results of all sampling operations are counted to obtain the average value, i.e. the target value of the power distribution network reliability index. In this experiment, common sampling method and average and scattered sampling method were used for reliability analysis of the same network. The specific experimental data of multiple experiments is shown in Table 1.

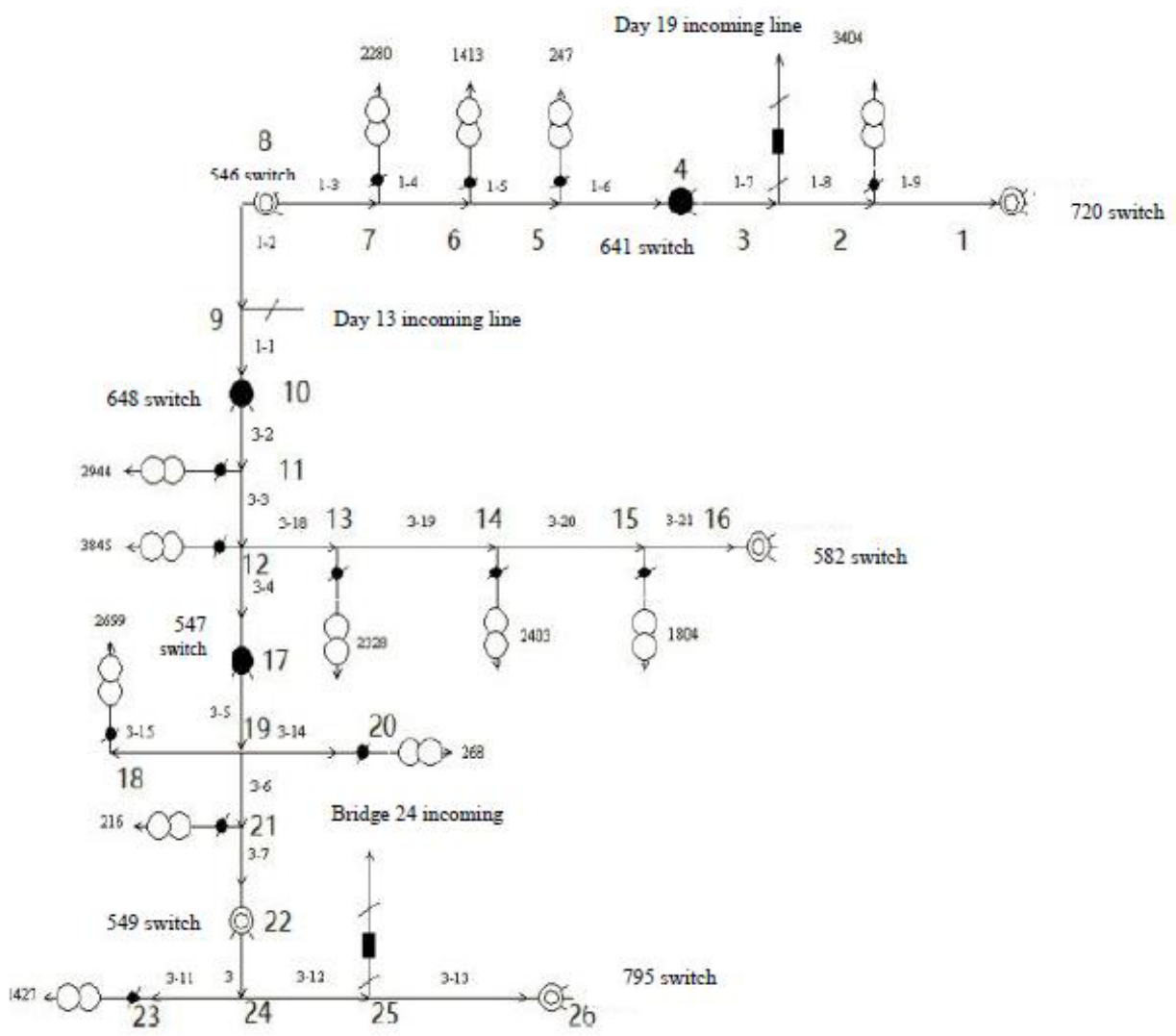

Fig. 2 Power Distribution Network Structure 
Table 1 Reliability Calculation Results of Power Distribution Network

\begin{tabular}{|l|l|c|c|c|c|c|c|}
\hline & \multicolumn{1}{|c|}{ SUM } & SAIFI & SAIDI & CAIDI & ASAI & ENS & Time \\
\hline $\begin{array}{c}\text { Ordinary } \\
\text { sampling }\end{array}$ & 1773 & 0.167118535 & 0.880575877 & 5.269169432 & 0.999899478 & 2.31458926 & 600.2812500 \\
\hline $\begin{array}{c}\text { Ordinary } \\
\text { sampling }\end{array}$ & 1802 & 0.174769591 & 0.884536473 & 5.061157757 & 0.999899026 & 2.357938961 & 600.2812500 \\
\hline $\begin{array}{l}\text { Ordinary } \\
\text { sampling }\end{array}$ & 1811 & 0.170485244 & 0.859122941 & 5.039280361 & 0.999901927 & 2.281123884 & 600.2656250 \\
\hline $\begin{array}{c}\text { Ordinary } \\
\text { sampling }\end{array}$ & 5523 & 0.168725837 & 0.835373268 & 4.951069048 & 0.999904638 & 2.238578926 & 1800.2656250 \\
\hline $\begin{array}{c}\text { Ordinary } \\
\text { sampling }\end{array}$ & 21447 & 0.169801149 & 0.837376009 & 4.931509677 & 0.999904409 & 2.264007962 & 7200.2812500 \\
\hline $\begin{array}{c}\text { Uniform } \\
\text { sampling }\end{array}$ & 6345277 & 0.166269346 & 0.830837862 & 4.996939506 & 0.999905156 & 2.255971281 & 1800.0156250 \\
\hline $\begin{array}{c}\text { Uniform } \\
\text { sampling }\end{array}$ & 6390384 & 0.166207687 & 0.830577778 & 4.99722841 & 0.999905185 & 2.255046977 & 1800.1406250 \\
\hline $\begin{array}{l}\text { Uniform } \\
\text { sampling }\end{array}$ & 198496449 & 0.166114804 & 0.830629097 & 5.000331562 & 0.999905179 & 2.254982628 & 55000.0156250 \\
\hline
\end{tabular}

In the reliability assessment, statistics is made on average power outage frequency index of the system, average power outage time, users' average power outage time and expected power shortage indicator. SUM value means the time duration of Monte Carlo simulation usage, which is in unit of year. The last time indicates program operation time in unit of second.

By analyzing the data obtained, it can be seen that simulation speed of both Monte Carlo method using uniform sampling method and Monte Carlo method using ordinary sampling method increases as the simulation period increases. In addition, there is a large gap in the indicators between the minimum simulation period and the maximum simulation period. For closer years, the simulated indicators are also closer. Comparison reveals that the average and scattered sampling method not only performs more simulations in a shorter time, but also greatly improves result accuracy. In terms of computational efficiency, average and scattered sampling method has been improved by about two orders of magnitude compared with ordinary sampling method, and the accuracy has also been increased by an order of magnitude.

In terms of reliability, the system has extremely high reliability. The index meets the reliability guidelines for Shanghai power distribution network that the reliability of full-caliber power grid should be above $99.99 \%$, and the reliability of city center should be above $99.999 \%$. It also meets the national power industry requirement that the power supply reliability in the region should be above or equal to $99.990 \%$. Also, the accuracy grade far exceeds these requirements. The average power outage time of the system is $0.16 \sim 0.17$ times, which is at a relatively high level compared with that in other cities. However, the user's power outage time is as much as 5 hours per household per year, exceeding the prescribed $52 \mathrm{~min}$.

\section{Factors that Affect Reliability and Reliability Improvement}

\subsection{Factors Affecting Reliability}

The factors affecting reliability of power supply can be classified as equipment factors, network structure, and external factors.

1. Equipment factors mean the power supply of the power grid is affected for the reason of the equipment itself. When new equipment is put into operation, there will be a run-in period. The failure rate will gradually decrease after the equipment operates for a period of time. Then, the equipment will enter a period of stability when the failure rate will be reduced to a minimum. However, the failure rate will gradually increase when it approaches the service life.

2. In terms of network structure, factors such as line segmentation and connection may affect line maintenance, which will greatly influence the control of the outage range. At the same time, factors such as wire diameter, power supply radius range, switch reliability and whether the cable ring network is connected in series to the overhead network will also affect power supply reliability.

3. The power outage event means the power company needs to cut off a part of the load when there is need to operate the relevant equipment according to the actual operation of the power grid. When it is scheduled in advance according to a reasonable plan, the power outage scope can be reduced and the power outage duration can be shortened so that normal use of electricity by neighboring users will not be affected as much as possible In addition, power supply may be rationed based on the operation condition when the current power supply capacity cannot meet the requirements but outage is unnecessary.

4. External factors are mainly divided into climate factors and human factors.

\subsection{Reliability improvement}

The power distribution reliability of power distribution network can be improved by taking the following measures based on the specific grid conditions:

1. Improve equipment reliability: The power grid is composed of various electrical equipment. The power grid will be more reliable in case of reliable equipment, and equipment reliability is a necessary condition for reliable power grid. According to statistical values of equipment reliability parameters (such as the average 
number of failures per year, the repair time required for each failure, etc.) and impact analysis of equipment failure on power grid reliability, it is possible to judge which equipment has poor reliability and which equipment greatly influences power grid reliability, so that management and maintenance of such equipment can be strengthened, or replacement can be made.

2. Change or strengthen the grid structure: a strong grid structure can effectively reduce the average time of scheduled and fault outage, which serves as the basis for improving power supply reliability. Grid structure irrationality will affect the power supply reliability of the grid to a large extent. In the power grid planning and construction, it is necessary to take into account the path which the corresponding load can be transferred to after equipment failure from the aspect of connection mode to improve power supply reliability of the power grid. At different load levels, the grid operates in different ways and its power supply reliability may not be identical. For different load levels, the power supply reliability level can be improved by changing operation mode of the power grid.

3. Change the equipment maintenance mode: The fault power outage maintenance or scheduled power outage maintenance greatly affects the power supply reliability during the maintenance period. By strengthening the maintenance efforts or taking hot line maintenance operation mode, equipment fault maintenance time and planned maintenance time can be shortened. By adopting condition based maintenance, the scheduled maintenance times that require power outages will be less.

4. Invest in distribution automation equipment: Input of distribution automation equipment can greatly shorten the fault point search and isolation time, rapidly restore the load power supply in non-fault areas, reduce users' power outage time and power outage range, and significantly improve power supply reliability of the power grid.

\section{Conclusion}

With the increased complexity and scale of power system grid structure, some traditional reliability calculation methods have been unable to meet the needs of modern distribution network reliability assessment due to the limitations in calculation accuracy and speed. Monte Carlo method should be used more as its accuracy is free from influence of power system scale change and its mathematical model is relatively simple. This study evaluated power distribution network reliability using improved Monte Carlo method, sampled the state of power distribution network system with analysis and calculation made. Statistical analysis was made on power system evaluation indicators, and the evaluation value was made to converge to the truth value of the value to be evaluated when there were ample samples. The results show that the improved Monte Carlo method with average and scattered sampling improves the speed and accuracy of reliability assessment, saves computation time, and provides a good direction for future reliability assessment of power distribution networks.

\section{References}

1. Li Zhimin, Li Weixing. Fault Traversal Algorithm for System Reliability Assessment Based on Radial Distribution[J]. Automation of Electric Power Systems, 2002, 26(2): 53-56.

2. Li Weixing, Li Zhimin, Liu Yingchun. Reliability Assessment of Complex Radial Distribution System[J]. Proceedings of the CSEE, 2003, 23(3): 69-73.

3. Xu Zhenxia, Zhou Jiangxin. Improved Fault Traversal Method for Reliability Assessment of Complex Distribution Networks [J]. Power System Technology, 2005, 29(14):64-67

4. Bie Zhaohong, Wang Xifan. Reliability Analysis of Power Distribution System [J]. Electric Power, 1997(5):10-13.

5. Xie K, Zhou J, Billinton R. Reliability Evaluation Algorithm for Complex Medium Voltage Electrical Distribution Networks Based on The Shortest Path[J]. IEE Proceedings Generation, Transmission and Distribution, 2004,150(6):686-690.

6. ÖZdemirA, Caglar R, Mekic F. A New Active Failure Simulation Approach for Distribution System Reliability Assessment[C].International Conference on Power. System Technology, 1998. Proceedings. Powercon. IEEE, 1998:237-240 vol. 1.

7. Wang $\mathrm{P}$, Billinton R, GoelL. Unreliability Cost Assessment of an Electric Power System Using Reliability Network Equivalent Approach [J]. IEEE Power Engineering Review, 2007, 22(7):58-58.

8. Wei Zhinong, Zhou Fengwei, Xiao Chuanling, et al. Reliability Evaluation Algorithm for Complex Medium Voltage Distribution Network Analysis Based on Simplified Network Model[J]. Power System Technology, 2006, 30(15):72-75 .

9. Goel L. Monte Carlo Simulation-Based Reliability Studies of a Distribution Test System[J].Electric Power Systems Research, 2000, 54(1):55-65.

10. Huda ASN, Živanović R. Accelerated Distribution Systems Reliability Evaluation by Multilevel Monte Carlo Simulation: Implementation of Two Discretisation Schemes[J]. LET Generation Transmission \& Distribution, 2017, 11(13):33973405.

11. Wan Guocheng, Ren Zhen, Wu Risheng, et al. Application of Hybrid Method in Reliability Assessment of Complex Distribution Network [J].Proceedings of the CSEE, 2004, 24(9):92-98. 